\title{
The Outer Halos of Early-Type Galaxies
}

\author{
Ortwin Gerhard $^{1}$, Magda Arnaboldi ${ }^{2}$ and Alessia Longobardi ${ }^{1}$ \\ ${ }^{1}$ Max-Planck-Institut für extraterrestrische Physik, Postfach 1312, Giessenbachstr. 1, 85741 \\ Garching, Germany. email: gerhard@mpe.mpg.de, alongobardi@mpe.mpg.de \\ ${ }^{2}$ European Southern Observatory, Karl-Schwarzschild-Strasse 2, D-85748 Garching, Germany. \\ e-mail: marnabol@eso.org
}

\begin{abstract}
The outer halos of massive early-type galaxies (ETGs) are dark matter dominated and may have formed by accretion of smaller systems during galaxy evolution. Here a brief report is given of some recent work on the kinematics, angular momentum, and mass distributions of simulated ETG halos, and of corresponding properties of observed halos measured with planetary nebulae (PNs) as tracers. In the outermost regions of the Virgo-central galaxy M87, the PN data show that the stellar halo and the co-spatial intracluster light are distinct kinematic components.
\end{abstract}

\section{Stellar and dark matter halos in simulated ETGs}

Wu et al. (2014) studied the luminous and dark matter halos in 42 simulated early-type galaxies (ETGs) with stellar masses from $2.0 \times 10^{10} \mathrm{M}_{\odot} \mathrm{h}^{-1}$ to $3.4 \times 10^{11} \mathrm{M}_{\odot} \mathrm{h}^{-1}$. These simulated galaxies were obtained from N-body/hydrodynamical zoom re-simulations of selected halos from a dark matter cosmological simulation, including a model for star formation and feedback (Oser et al. 2010, 2012).

The dark matter halo density profiles of the simulated ETGs follow simple power-law models, with flat dark matter circular velocity curves (CVCs) for lower mass systems and rising CVCs for high-mass halos, corresponding to slightly falling to flat total CVCs respectively. The dark matter fractions of these systems are in the range 15-30\% at the stellar half-mass radius $R_{e}$ and increase to $40-65 \%$ at $5 \mathrm{R}_{\mathrm{e}}$. The short axes of simulated galaxies and their host dark matter halos are well-aligned and their (short-to-long) axis ratios are correlated.

Line-of-sight (LOS) velocity fields obtained with a time-smoothing technique out to large radii (see examples in Fig. 1) show that the rotation properties at small and large radii are correlated. Radial profiles for the cumulative specific angular momentum parameter $\lambda(R)$ are nearly flat or slightly rising, with values in $[0.06,0.75]$ from $2 \mathrm{R}_{\mathrm{e}}$ to $5 \mathrm{R}_{\mathrm{e}}$. A few cases show local maxima in $|\bar{v}| / \sigma(R)$.

Many of the properties of these simulated ETGs agree remarkably well with observations of ETGs at large radii, such as briefly summarized in the next section.

\section{Kinematics, angular momentum, and dark matter mass in ETG halos from PNs}

Planetary nebulae (PNs) are excellent tracers for the kinematics in the faint outskirts of ETGs where kinematic measurements from integrated light are no longer possible. Various techniques have been implemented to measure radial velocities from their bright [OIII] ג5007Å line (e.g., Hui et al. 1995; Arnaboldi et al. 1998; Méndez et al. 2001; McNeil et al. 2010). With the specially built Planetary Spectrograph (PN.S) instrument (Douglas et al. 2002), a survey of nearby $(D<20 \mathrm{Mpc})$ early-type galaxy halos in the Northern hemisphere has now been completed. The new sample expands on earlier results based 

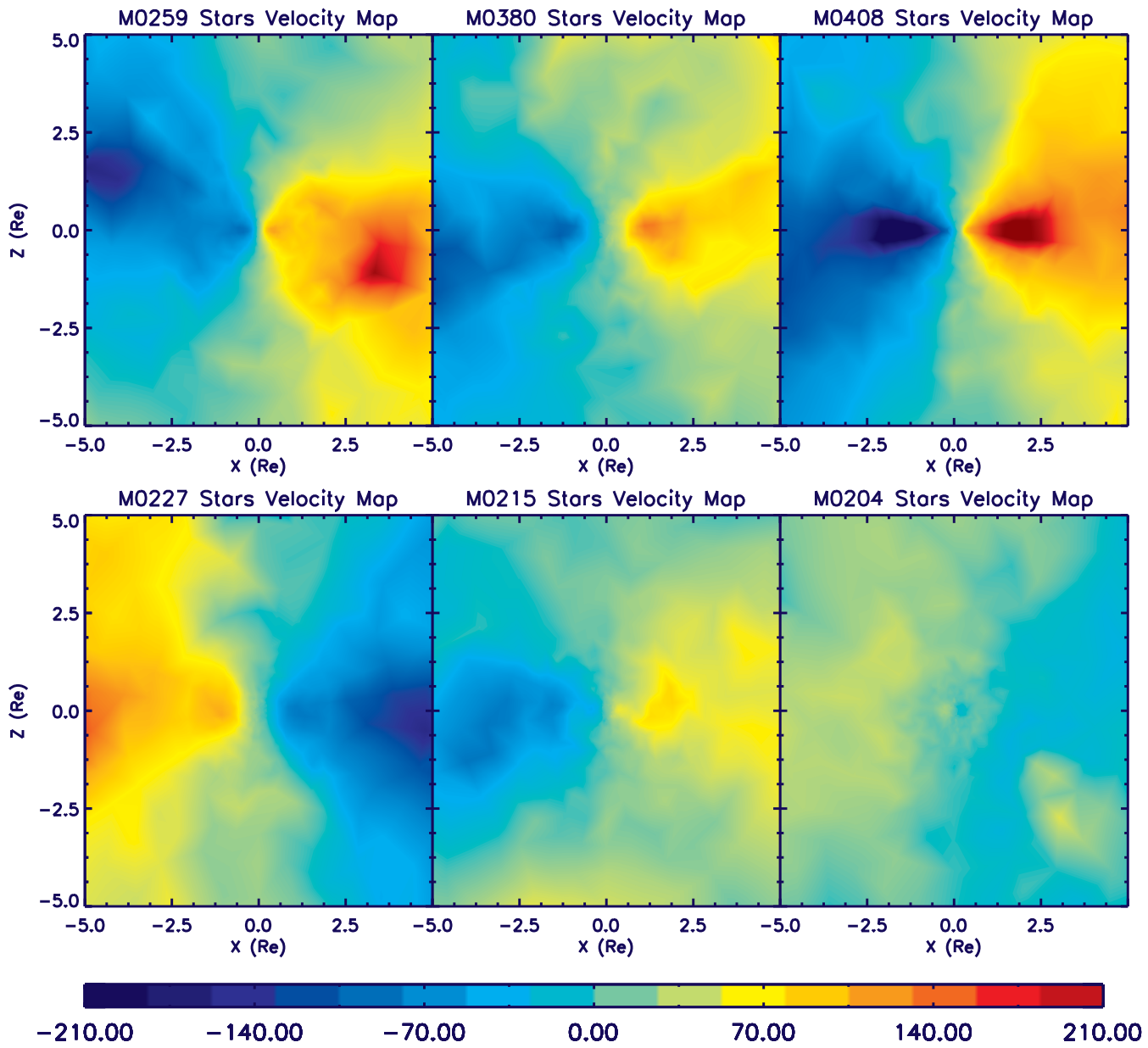

Figure 1. Edge-on mean LOS velocity maps for the stellar components of 6 selected model galaxies. The upper panels show three simulated galaxies with large scale rotation; the galaxy in the top right panel has a strong disk component from a gas rich major merger. The lower panels show three simulated galaxies with a dissipationless merging history. The galaxy in the bottom right panel shows weak major and minor axis rotation; it has a formation history with many minor mergers. From Wu et al. (2014).

on 6 lenticulars and 13 ellipticals (Coccato et al. 2009; Cortesi et al. 2013), to 11 S0s and 30 ellipticals with extended kinematics measured from $2-10 R_{e}$.

From the PN data one can measure the specific angular momentum out to these large radii (Fig. 2, from Cortesi et al. 2013) and the frequency of angular momentum misalignment between the inner region and the outer halo. One interesting, still not fully understood result is the apparent dichotomy between galaxies with quasi-Keplerian, steeply falling, velocity dispersion profiles and galaxies with nearly flat velocity dispersion profiles (Coccato et al. 2009). Dynamical modeling of a few of the quasi-Keplerian systems with the NMAGIC made-to-measure method (de Lorenzi et al. 2007) has shown a strong mass-anisotropy degeneracy (de Lorenzi et al. 2008, 2009), with the range of best dark matter halo profiles (Fig. 3; Morganti et al. 2013) consistent with those of the lower mass, simulated ETGs of Wu et al. (2014); see also Deason et al. (2012). By contrast, for massive ETGs with nearly flat dispersion profiles the inferred total CVCs are nearly 


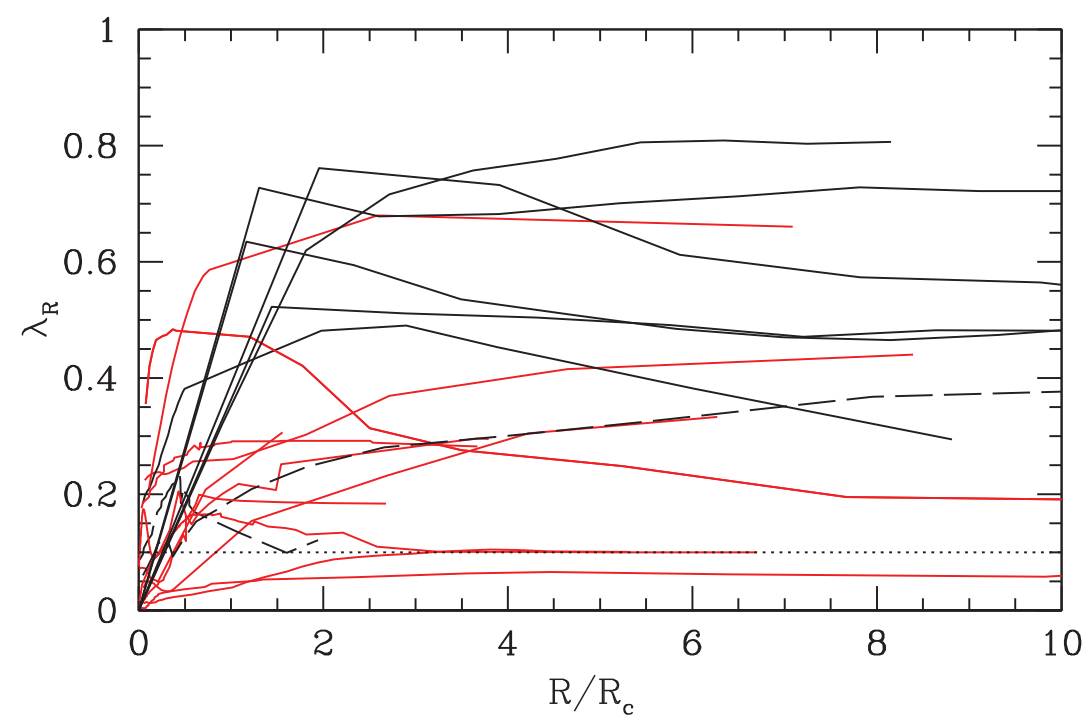

Figure 2. The $\lambda_{R}$ parameter measuring enclosed specific angular momentum as a function of radius, for a sample of early-type galaxies. Black solid lines are S0s from Cortesi et al. (2013), black dashed lines are S0s from Coccato et al. (2009), and red lines are ellipticals from Coccato et al. (2009). The dotted line shows the suggested separation between slow and fast rotators (Emsellem et al. 2007). From Cortesi et al. (2013).

constant with radius (e.g., Das et al. 2011). Full analysis of the PN.S survey data is still on-going.

\section{Distinct stellar halo and intracluster light components in the outskirts of the giant Virgo ETG M87}

Brightest cluster galaxies (BCGs) have extended halos often embedded in diffuse intracluster light (ICL; e.g., Gonzalez et al. 2005). The ICL consists of stars moving in the cluster potential, whereas halo stars would be defined as bound to the central galaxy. However, if the BCG is located in the cluster center, it is not clear whether a clear distinction between halo and ICL is generally possible (Dolag et al. 2010; Cooper et al. 2013). An interesting case is NGC 3311 in the Hydra I cluster, where the very rapid transition of the velocity dispersion profile to cluster values suggests a continuous transition from halo to ICL (Ventimiglia et al. 2010).

Doherty et al. (2009) first investigated the transition from halo to ICL in the outermost regions of the Virgo cluster. With a small sample of PNs, they found no halo PNs beyond $150 \mathrm{kpc}$ radius, and a rapidly decreasing velocity dispersion profile towards this boundary, while ICL PNs with large velocity dispersion where seen at similar radii. Based on a large sample of PN candidates indentified through on/off-band imaging (Longobardi et al. 2013), and kinematic follow-up with the VLT FLAMES instrument (Longobardi et al. 2014), it was possible to separate PNs from the M87 halo and ICL with a robust kinematic technique. Fig. 4 shows that both components coexist at similar radii with different density profiles. The relative abundance of PNs per unit bolometric luminosity and the PN luminosity function furthermore suggest that the outer M87 halo and the ICL in the Virgo core are different stellar populations. 


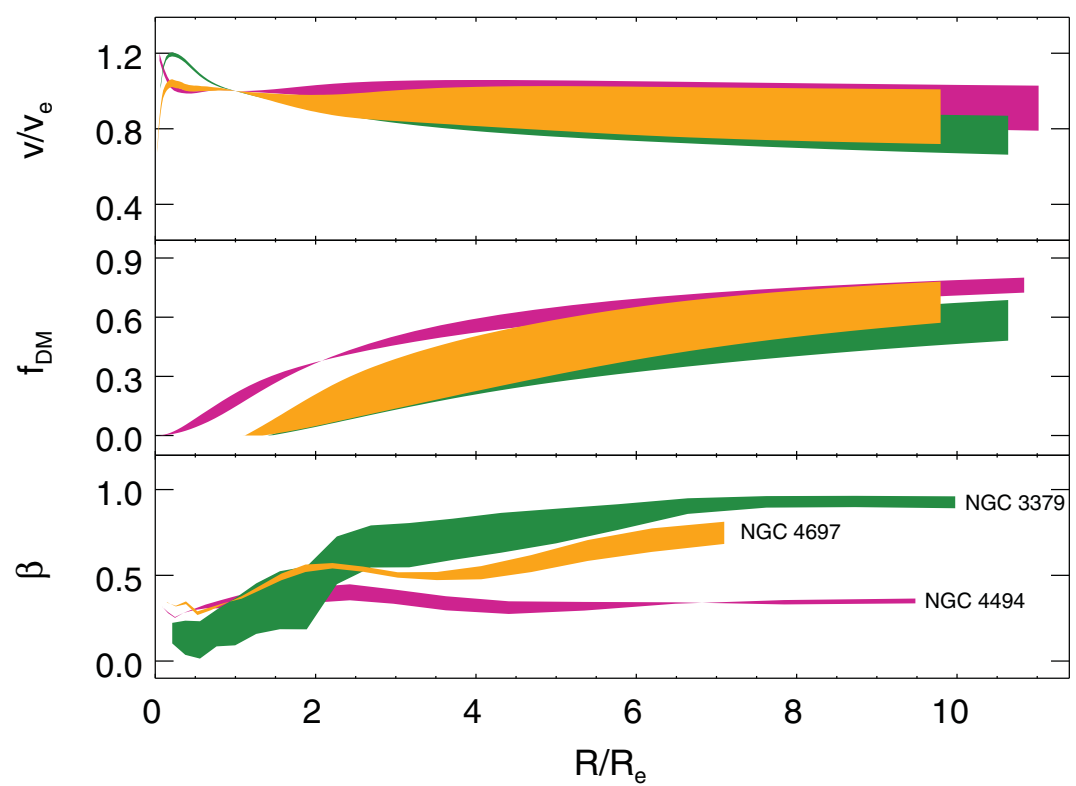

Figure 3. Radial profiles of circular velocity normalized by its value at $1 R_{\mathrm{e}}$ (top), dark matter fraction (middle), and anisotropy parameter (bottom), for the range of valid NMAGIC models obtained fitting the data of NGC 4494 (violet, Morganti et al. 2013), NGC 4697 (orange, de Lorenzi et al. 2008), and NGC 3379 (green, de Lorenzi et al. 2009). From Morganti et al. (2013).

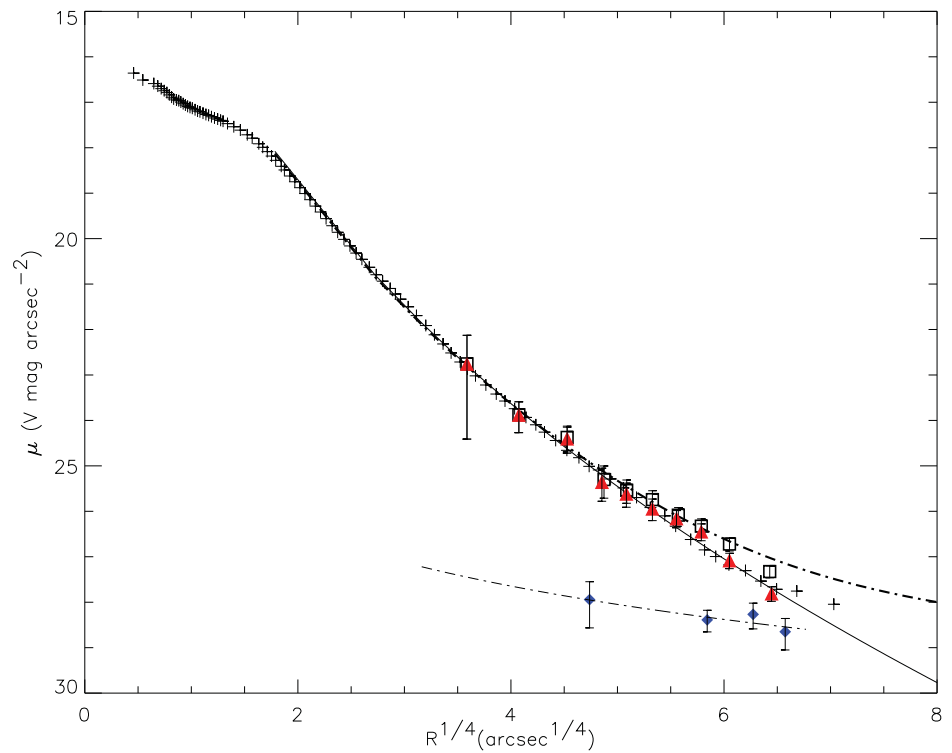

Figure 4. The logarithmic PN number density profile of the M87 halo (red triangles) and ICL PNs (blue diamonds), corrected for incompleteness as a function of the distance from the M87 centre. For comparison, the surface brightness profile from Kormendy et al. (2009) is shown as crosses. The ICL density decreases towards larger radii as $\mathrm{I}_{\mathrm{ICL}} \propto \mathrm{R}^{-0.4}$ (dashed blue line). Black squares show the composite halo plus ICL PN number density profile, well-modelled by the two component model (dot-dashed black line, see Longobardi et al. (2014) for more details). Error bars account for $1 \sigma$ uncertainties from counting statistics. From Longobardi et al. (2014). 


\section{References}

Arnaboldi, M., et al. 1998, ApJ, 507, 759

Coccato, L., et al. 2009, MNRAS, 394, 1249

Cooper, A. P., et al. 2013, MNRAS, 434, 3348

Cortesi, A., et al. 2013, MNRAS, 432, 1010

Das, P., et al. 2011, MNRAS, 415, 1244

de Lorenzi, F., Debattista, V. P., Gerhard, O., \& Sambhus, N. 2007, MNRAS, 376, 71

de Lorenzi, F., et al. 2008, MNRAS, 385, 1729

-. 2009, MNRAS, 395, 76

Deason, A. J., Belokurov, V., Evans, N. W., \& McCarthy, I. G. 2012, ApJ, 748, 2

Doherty, M., et al. 2009, A\& $A, 502,771$

Dolag, K., Murante, G., \& Borgani, S. 2010, MNRAS, 405, 1544

Douglas, N. G., et al. 2002, PASP, 114, 1234

Emsellem, E., et al. 2007, MNRAS, 379, 401

Gonzalez, A. H., Zabludoff, A. I., \& Zaritsky, D. 2005, ApJ, 618, 195

Hui, X., Ford, H. C., Freeman, K. C., \& Dopita, M. A. 1995, ApJ, 449, 592

Kormendy, J., Fisher, D. B., Cornell, M. E., \& Bender, R. 2009, ApJS, 182, 216

Longobardi, A., et al. 2013, A\&A A, 558, A42

Longobardi, A., Arnaboldi, M., Gerhard, O., \& Hanuschik, R. 2014, A\&A, submitted

McNeil, E. K., et al. 2010, A\&3A, 518, A44

Méndez, R. H., et al. 2001, ApJ, 563, 135

Morganti, L., et al. 2013, MNRAS, 431, 3570

Oser, L., Naab, T., Ostriker, J. P., \& Johansson, P. H. 2012, ApJ, 744, 63

Oser, L., et al. 2010, ApJ, 725, 2312

Ventimiglia, G., Gerhard, O., Arnaboldi, M., \& Coccato, L. 2010, A\&3A, 520, L9+

Wu, X., et al. 2014, MNRAS, 438, 2701 